\title{
Long-term amiodarone therapy and the risk of complications after cardiac surgery: Results from the Canadian Amiodarone Myocardial Infarction Arrhythmia Trial (CAMIAT)
}

\author{
Eugene Crystal, $\mathrm{MD}^{\mathrm{a}, \mathrm{e}}$ \\ Shoshanah Kahn, MDa \\ Robin Roberts, MTech ${ }^{b}$ \\ Kevin Thorpe, MMath ${ }^{\mathrm{b}}$ \\ Michael Gent, DSc ${ }^{\mathrm{b}}$ \\ John A. Cairns, MD \\ Paul Dorian, $\mathrm{MD}^{\mathrm{d}}$ \\ Stuart J. Connolly, MDa \\ On behalf of the CAMIAT investigators
}

See related editorial on page 463.
From the Departments of Medicine ${ }^{\mathrm{a}}$ and Clinical Epidemiology and Biostatistics, ${ }^{\text {b }}$ Faculty of Health Sciences, McMaster University, Hamilton, Ontario, the Department of Medicine, University of British Columbia, Vancouver, British Columbia, ${ }^{\mathrm{c}}$ and the Department of Medicine, University of Toronto, Toronto, Ontario, ${ }^{\mathrm{d}}$ Canada, and the Department of Cardiology, Faculty of Health Sciences, Ben-Gurion University, Beer-Sheva, Israel. ${ }^{e}$

Received for publication March 17, 2002; revisions requested April 16, 2002; revisions received June 24, 2002; accepted for publication Aug 6, 2002.

Address for reprints: Eugene Crystal, MD, 237 Barton St E, Arrhythmia Service, Hamilton General Hospital, Hamilton, Ontario L8L2X2,Canada(E-mail:crystal@bgumail. bgu.ac.il).

J Thorac Cardiovasc Surg 2003;125:633-7

Copyright $(\odot 2003$ by The American Association for Thoracic Surgery

$0022-5223 / 2003 \$ 30.00+0$

doi: $10.1067 / \mathrm{mtc} .2003 .9$
Objective: This study was undertaken to determine the association between amiodarone therapy and risk of complications of cardiac surgery in patients in the randomized placebo-controlled, double-blind Canadian Amiodarone Myocardial Infarction Arrhythmia Trial.

Methods: Prospectively collected data regarding postoperative complications in 82 patients who underwent cardiac surgery during Canadian Amiodarone Myocardial Infarction Arrhythmia Trial participation were analyzed; 36 patients were randomly assigned to receive amiodarone and 46 were assigned to receive placebo. Of the patients randomly assigned to receive amiodarone, 24 patients continued amiodarone treatment to within 7 days of the operation (active amiodarone group) and 12 patients had the amiodarone discontinued at least 7 days before the operation (discontinued amiodarone group).

Results: The baseline characteristics of the three groups were similar. The risks of ventricular fibrillation, atrial fibrillation, and respiratory complications were similar. The risk of requiring an intra-aortic balloon pump was significantly increased by amiodarone (34.8\% vs $16.7 \%$ vs $8.7 \%$ for active amiodarone, discontinued amiodarone, and placebo groups, respectively, $P=.024)$. There was no significant difference in the use of temporary pacing. Neither the mean duration of stay in the intensive care unit nor the 7- and 30-days mortalities were affected by amiodarone.

Conclusions: Patients receiving long-term amiodarone treatment after myocardial infarction had a higher rate of intra-aortic balloon use after cardiac surgery. There was no increased risk of pulmonary complications, need for pacing, or death.

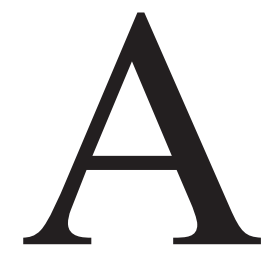

miodarone is the most widely used antiarrhythmic drug, and about $2 \%$ of patients referred for cardiac surgery are receiving long-term amiodarone therapy. ${ }^{1}$ This agent has several well-described sideeffects that could increase postoperative risk, and concern has been raised in several studies regarding the safety of cardiac surgery for patients receiving long-term amiodarone. Small series and case-control studies ${ }^{1-8}$ have raised concerns about the safety of amiodarone therapy for patients undergoing cardiac surgery. 
In these reports three adverse effects have been specifically associated with cardiac surgery in patients receiving long-term amiodarone therapy: pulmonary complications related to adult respiratory distress syndrome, ${ }^{2-4,6,7}$ prolonged hypotension related to resistant peripheral vasodilatation or low cardiac output,-9 and bradycardia requiring pacing. ${ }^{8-10}$ Although the adverse effects reported may have been due to amiodarone, the risk of postoperative complications from amiodarone may have been overestimated because patients who receive amiodarone often have advanced heart disease, and none of these studies had proper control groups. The observed adverse effects thus may have been related to the severe underlying cardiac disease rather than to amiodarone therapy itself. Indeed, in one case-control study ${ }^{1}$ no significant increase in complications was found in the amiodarone-treated group relative to the control group when multivariate analysis was used to account for the severity of underlying medical conditions. No randomized controlled data have been reported evaluating the perioperative risk of long-term amiodarone therapy for patients undergoing cardiac surgery.

The Canadian Amiodarone Myocardial Infarction Arrhythmia Trial (CAMIAT) was a randomized, double-blind, placebo-controlled trial of amiodarone among survivors of myocardial infarction at risk for arrhythmic death. ${ }^{11}$ To determine whether long-term amiodarone therapy increases the perioperative risk of cardiac surgery, adverse effect data related to cardiac surgery were collected prospectively for patients who participated in CAMIAT. This report compares the risk of postoperative complications related to cardiac surgery in CAMIAT patients randomly assigned in a double-blinded fashion to receive either amiodarone or placebo.

\section{Methods}

\section{Eligibility of Patients}

The eligibility criteria for CAMIAT have been published previously elsewhere. ${ }^{11}$ In brief, patients were eligible if they met the following criteria: (1) myocardial infarction within the previous 6 to 45 days, (2) 24-hour Holter monitoring showing $\geq 10$ ventricular premature beats/hour or at least one run of ventricular tachycardia, and (3) age greater than 19 years. The study was approved by local institutional review boards in all participating centers.

\section{Treatment and Follow-up}

Patients received an oral dose of $10 \mathrm{mg} /(\mathrm{kg} \times \mathrm{d}$ ) of amiodarone or placebo for 2 weeks, followed by $400 \mathrm{mg}$ daily, except in those patients less than $60 \mathrm{~kg}$ in weight or older than 75 years, for whom the dose was reduced to $300 \mathrm{mg}$ daily. Patients were reassessed at both months 4 and 8 by 24-hour Holter monitor. If arrhythmia suppression was observed, the dose was further reduced by 100 $\mathrm{mg} / \mathrm{d}$ to a potential minimum dose of $200 \mathrm{mg} / \mathrm{d}$, or $200 \mathrm{mg} / \mathrm{d} 5$ $\mathrm{d} /$ wk for those older than 75 years or less than $60 \mathrm{~kg}$ in weight.

\section{Cardiac Surgery in CAMIAT Patients}

The CAMIAT protocol specified that study patients who underwent elective heart surgery should stop study medication 7 days before the surgical date and that the medication be resumed after surgery once the patient was hemodynamically stable. Among the 1202 patients participating in the CAMIAT, surgical complication data were available for 87 patients who underwent cardiac surgery during the study (7.2\%). Among those 87 patients, 5 had had the study medication permanently discontinued more than 3 months before the operation, and they were not included in this analysis.

Of the remaining 82 patients, 46 had been randomly assigned to the placebo group and 36 had been assigned to the amiodarone group. In the amiodarone treatment group 24 patients were still receiving active medication within 7 days of surgery (active amiodarone group), whereas 12 had discontinued the study medication at least 7 days before the surgery (discontinued amiodarone group). The mean maintenance dose of amiodarone (among those patients who were receiving amiodarone) was $353 \pm 84 \mathrm{mg}$. The median number of days that amiodarone was stopped before the surgery (discontinued amiodarone group) was 15 days (interquartile range 11-41 days).

\section{Postoperative Outcome Events in Study Patients Undergoing Cardiac Surgery}

The postoperative period during which the adverse events were recorded was defined as beginning at the time of exit from the operating room until postoperative day 7 . The total number of days in the intensive care unit was noted for all patients. Only electrocardiographically documented postoperative arrhythmias were included. These included asystole of more than 4 seconds, complete atrioventricular block, new atrial fibrillation (lasting $>30$ minutes), and resuscitated ventricular fibrillation. Postoperative uses of temporary pacing, counter pulsation intra-aortic balloon pump, or inotropic drugs were recorded for all patients, and the total time in hours that such therapies were required was calculated as a period from the first beginning of the treatment to the last discontinuation. All cardiovascular, pulmonary, and other complications were recorded. The 7- and 30-day mortalities were recorded.

\section{Statistics}

Comparisons among the three groups used the Fisher exact test for categoric variables and the Kruskal-Wallis test for continuous variables.

\section{Results}

\section{Baseline Characteristics}

The baseline demographic and clinical characteristics of the patients are presented in Table 1. There were no differences in baseline patient characteristics with regard to mean age; previous (to index) myocardial infarction; previous history of pulmonary edema, stroke, chronic respiratory disease, or diabetes; or previous cardiac surgery. The types of surgical procedures performed were similar between groups, with most patients undergoing coronary artery bypass grafting. There was no difference in the preoperative use of $\beta$-blockers, diltiazem, or verapamil. 
TABLE 1. Baseline demographic and clinical characteristics

\begin{tabular}{|c|c|c|c|c|}
\hline \multirow[b]{2}{*}{ Patient characteristics } & \multicolumn{2}{|c|}{ Amiodarone $(n=36)$} & \multirow[b]{2}{*}{$\begin{array}{l}\text { Placebo } \\
(n=46)\end{array}$} & \multirow[b]{2}{*}{$P$ valuet } \\
\hline & $\begin{array}{c}\text { Active } \\
\text { amiodarone } \\
(\mathbf{n}=24)\end{array}$ & $\begin{array}{l}\text { Discontinued } \\
\text { amiodarone* } \\
\qquad(\mathbf{n}=12)\end{array}$ & & \\
\hline Age $(y$, mean $\pm S D)$ & $61.2 \pm 9.0$ & $61.4 \pm 8.8$ & $58.8 \pm 10.4$ & .523 \\
\hline Multiple myocardial infarctions (No.) & $7(29.2 \%)$ & $5(41.7 \%)$ & $23(50 \%)$ & .245 \\
\hline Previous pulmonary edema (No.) & $7(29.2 \%)$ & $3(25 \%)$ & $11(23.9 \%)$ & .937 \\
\hline Stroke (No.) & 0 & 0 & $4(8.7 \%)$ & .354 \\
\hline Chronic respiratory disease (No.) & $3(12.5 \%)$ & $2(16.7 \%)$ & $3(6.5 \%)$ & .391 \\
\hline Diabetes mellitus (No.) & $6(25.0 \%)$ & $4(33.3 \%)$ & $9(19.6 \%)$ & .581 \\
\hline Previous heart surgery (No.) & $1(4.2 \%)$ & $2(16.7 \%)$ & $3(6.5 \%)$ & .340 \\
\hline \multicolumn{5}{|l|}{ Type of index cardiac surgery (No.) } \\
\hline Coronary artery bypass grafting & $24(100.0 \%)$ & $12(100.0 \%)$ & $45(97.8 \%)$ & $>.999$ \\
\hline Aortic valve replacement & 0 & $1(8.3 \%)$ & $1(2.2 \%)$ & .356 \\
\hline Mitral valve replacement & 0 & 0 & $2(4.4 \%)$ & .668 \\
\hline Aneurysm resection & $1(4.2 \%)$ & $1(8.3 \%)$ & $5(10.9 \%)$ & .858 \\
\hline Other & 0 & $1(9.1 \%)$ & $2(4.4 \%)$ & .242 \\
\hline \multicolumn{5}{|l|}{ Medications within 24 hours (No.) } \\
\hline Verapamil & $1(5.6 \%)$ & 0 & $1(2.7 \%)$ & $>.999$ \\
\hline Diltiazem & $6(33.3 \%)$ & $3(42.9 \%)$ & $13(35.1 \%)$ & .861 \\
\hline$\beta$-Blocker & $12(66.7 \%)$ & $5(71.4 \%)$ & $28(75.7 \%)$ & .772 \\
\hline $\begin{array}{l}\text { Time between random assignment } \\
\text { and heart surgery ( } d \text {, median and } \\
\text { interquartile range) }\end{array}$ & $90(21-116)$ & $164(97-306)$ & $154(47-244)$ & .063 \\
\hline
\end{tabular}

${ }^{*}$ Amiodarone stopped 7 days before surgery per CAMIAT protocol.

$\dagger P$ value for comparison of three groups.

\section{Hospital Course and Complications}

The rates of postoperative asystole (Table 2) were similar in the three study groups: $4.2 \%$ of patients in the active amiodarone group, $8.3 \%$ of patients in the discontinued amiodarone group, and $4.4 \%$ of patients in the placebo group $(P=.79)$. The incidences of postoperative ventricular fibrillation were similar in the three study groups: $4.2 \%$ of patients in the active amiodarone group, $8.3 \%$ of patients in the discontinued amiodarone group, and $13.3 \%$ of patients in the placebo group $(P=.6)$. The incidences of atrial fibrillation were $16.7 \%$ in the active amiodarone group, $16.7 \%$ in the discontinued amiodarone group, and $24.8 \%$ in the placebo group $(P=.7)$. The rates for use of temporary pacing were $26.1 \%$ in the active amiodarone group, $25 \%$ in the discontinued amiodarone group, and $10.9 \%$ in the placebo group. The prespecified three-group comparison of three differences in need for temporary pacing had a $P$ value of .19; the $P$ value for the comparison of the combined amiodarone groups against placebo was also firmly nonsignificant at a $P$ value of .14. The mean durations of temporary pacing were $37.2 \pm 30.7$ hours, $7.3 \pm 6.0$ hours and $7.2 \pm 6.1$ hours for the active amiodarone, discontinued amiodarone, and placebo groups, respectively $(P=.12)$.

Intra-aortic balloon pumps were used by 10 study centers in 14 patients. Patients on amiodarone were significantly more likely to use intra-aortic balloon pump therapy (34.8\% in the active amiodarone group, $16.7 \%$ in the discontinued amiodarone group, and $8.7 \%$ in the placebo group, $P=.03$ ). The indications for balloon pump therapy were not specifically collected. In terms of duration of the support, the data from the discontinued amiodarone group $(\mathrm{n}=2)$ were unavailable, but no significant difference was found between the active amiodarone and placebo groups $(93 \pm 171$ hours in active amiodarone group vs $42 \pm 61$ hours in placebo group, $P=.67$ ).

The rates of catecholamine drug use were similar in the three study groups $(60.9 \%$ in the active amiodarone group, $50.0 \%$ in the discontinued amiodarone group, and $43.5 \%$ in the placebo group, $P=.37$ ). The duration of inotropic support was not significantly different among the groups $(P=.31)$.

Pulmonary complications were infrequent, and their rate did not differ among the study groups: $0 \%$ in the active amiodarone group, $16 \%$ in the discontinued amiodarone group, and $2 \%$ in the placebo group $(P=.4)$.

Mortalities and durations of intensive care unit stay are shown in Table 3. The mean postoperative intensive care unit stays were similar in the three study groups: $6.1 \pm 9.2$ days in the active amiodarone group, $2.8 \pm 1.6$ days in the discontinued amiodarone group, and $3.6 \pm 3.9$ days in the placebo group $(P=.41)$. The 7 -day mortality was $0 \%$ in the active amiodarone group, $8.3 \%$ in the discontinued amiodarone group, and $0 \%$ in the placebo group $(P=.15)$. 
TABLE 2. Postoperative complications

\begin{tabular}{|c|c|c|c|c|}
\hline \multirow[b]{2}{*}{ Complications } & \multicolumn{2}{|c|}{ Amiodarone $(n=36)$} & \multirow[b]{2}{*}{$\begin{array}{l}\text { Placebo } \\
(\mathrm{n}=46)\end{array}$} & \multirow[b]{2}{*}{$P$ value } \\
\hline & $\begin{array}{c}\text { Active } \\
\text { amiodarone* } \\
(n=24)\end{array}$ & $\begin{array}{l}\text { Discontinued } \\
\text { amiodarone } \\
\text { (n=12*) }\end{array}$ & & \\
\hline \multicolumn{5}{|l|}{ Arrhythmia } \\
\hline Asystole $>4 \mathrm{~s}$ (No.) & $1(4.2)$ & $1(8.3)$ & $2(4.4 \%)$ & .8 \\
\hline Ventricular fibrillation (No.) & $1(4.2)$ & $1(8.3)$ & $6(13.3 \%)$ & 6 \\
\hline New atrial fibrillation (No.) & $4(16.7)$ & $2(16.7)$ & $11(24.8 \%)$ & .7 \\
\hline Temporary pacing need (No.) & $6(26.1)$ & $3(25.0)$ & $5(10.9 \%)$ & .19 \\
\hline $\begin{array}{l}\text { Mean duration of temporary } \\
\text { postoperative pacing }(h \text {, mean } \pm S D)\end{array}$ & $37.2 \pm 30.7$ & $7.3 \pm 6.0$ & $7.2 \pm 6.1$ & $.117 \dagger$ \\
\hline \multicolumn{5}{|l|}{ Hemodynamic instability } \\
\hline Need for balloon pump (No.) & $8(34.8)$ & $2(16.7)$ & $4(8.7 \%)$ & .027 \\
\hline $\begin{array}{l}\text { Duration of pump support (h, } \\
\text { mean } \pm \text { SD) }\end{array}$ & $93.1 \pm 171.0$ & - & $32.5 \pm 43.2$ & $.669 \dagger$ \\
\hline Need for inotropic drugs (No.) & $14(60.9)$ & $6(50.0)$ & $20(43.5 \%)$ & .371 \\
\hline $\begin{array}{l}\text { Duration of inotropic drug support } \\
\text { (h, mean } \pm \text { SD) }\end{array}$ & $136.8 \pm 195.1$ & $30.3 \pm 34.4$ & $42.4 \pm 61.4$ & $.314 \dagger$ \\
\hline Pulmonary complications (No.) & 0 & $2(16.6)$ & $1(2.2 \%)$ & .4 \\
\hline
\end{tabular}

*Amiodarone stopped 7 days before surgery per CAMIAT protocol.

†Nonparametric value.

TABLE 3. Postoperative intensive care unit stay and mortality

\begin{tabular}{|c|c|c|c|c|}
\hline & \multicolumn{2}{|c|}{ Amiodarone $(n=36)$} & \multirow[b]{2}{*}{$\begin{array}{l}\text { Placebo } \\
(\mathrm{n}=46)\end{array}$} & \multirow[b]{2}{*}{$P$ value } \\
\hline & $\begin{array}{c}\text { Active } \\
\text { amiodarone } \\
(\mathrm{n}=24)\end{array}$ & $\begin{array}{l}\text { Discontinued } \\
\text { amiodarone* } \\
\quad(n=12)\end{array}$ & & \\
\hline $\begin{array}{l}\text { Postoperative intensive } \\
\text { care unit stay } \\
\text { (d, mean } \pm \text { SD) }\end{array}$ & $6.1 \pm 9.2$ & $2.8 \pm 1.6$ & $3.6 \pm 3.9$ & .412 \\
\hline 7-d mortality & $0 \%$ & $8.3 \%$ & $0 \%$ & .146 \\
\hline 30-d mortality & $4.2 \%$ & $8.3 \%$ & $2.2 \%$ & .548 \\
\hline
\end{tabular}

${ }^{*}$ Amiodarone stopped 7 days before surgery per CAMIAT protocol.

Thirty-day mortality rates were $4.2 \%, 8.3 \%$ and $2.2 \%$, respectively $(P=.55)$.

\section{Discussion}

These results from a randomized, double-blind, placebocontrolled trial support earlier observations that long-term amiodarone therapy increases postoperative risk of prolonged systemic hypotension requiring placement of an intra-aortic balloon pump. Unlike many previous reports, no significant increases were seen in risk of pulmonary complications or in bradycardia requiring pacing. Discontinuation of amiodarone treatment for about a week before the operation may decrease the risk of amiodarone-associated hypotension and the need for intra-aortic balloon pump placement.

The intra-aortic balloon pump is used to increase cardiac output and to manage systemic hypotension. Intractable vasodilatation leading to prolonged hypotension, with or without low cardiac output, has been previously reported postoperatively in patients receiving long-term amiodarone therapy. ${ }^{1,8,9,12}$ This is probably due to accentuation of one of amiodarone's known pharmacologic actions, sympathetic system blockade, which may result in profound vasodilatation. ${ }^{9,12}$ The use of vasopressor drugs in this study was somewhat more frequent among patients receiving amiodarone than those receiving placebo, but the difference were not statistically significant. The observed use of intra-aortic balloon pumping with amiodarone may be depend on simultaneous treatment with other drugs, such as $\beta$-blockers and angiotensin-converting enzyme inhibitors, which potentially depress the available compensatory mechanisms. ${ }^{12}$

Amiodarone is known to cause depression of sinus and atrioventricular node automaticity and conduction, and an increased use of perioperative pacemaker therapy in patients receiving amiodarone has been previously reported. ${ }^{8-10}$ The study groups were well balanced in the use of other drugs 
that affect the cardiac conduction system, such as $\beta$-blockers and calcium-channel blockers (Table 1). It appears that the bradycardia caused by amiodarone is not worsened in the perioperative state.

Long-term amiodarone therapy can cause a pulmonary interstitial infiltrate during. ${ }^{13}$ An acute adult respiratory distress syndrome occurring in immediate postoperative period has been attributed to amiodarone. ${ }^{2-7}$ On the other hand, some studies ${ }^{1,8}$ reported no significant increase in postoperative pulmonary complications among patients receiving long-term amiodarone therapy. Previous studies have varied widely in their estimates of the relative risk of development of adult respiratory distress syndrome with amiodarone, from no increase ${ }^{1}$ to a 5 -fold increase. ${ }^{4}$ In our study the risk of postoperative respiratory complications was small in all groups and was not increased by the long-term use of amiodarone. This finding suggests that the risk of amiodarone-induced pulmonary complications was overestimated in these previous nonrandomized reports.

The observed rate of postoperative atrial fibrillation was lower among patients receiving amiodarone, but the difference was not statistically significant (Table 2). Several studies have reported that amiodarone reduces atrial fibrillation both during long-term follow-up and when used prophylactically after heart surgery. ${ }^{14}$ It is likely that the relatively small number of patients in this analysis rendered it underpowered to detect a true difference.

Discontinuation of amiodarone before cardiac surgery for the median period of 2 weeks was associated with a reduction in amiodarone-associated increased need for intra-aortic balloon pumping. It was also associated with a trend toward a shorter period of temporary pacing. Although the CAMIAT protocol specified withdrawal of study drug 7 days before elective surgery, the actual decision to withdraw the drug was made by the investigators. Because amiodarone withdrawal before surgery was not randomly determined, differences between patients who had amiodarone withdrawn and those who did not may be due to patient selection. Nonetheless, the data from the analysis support the concept of withdrawing amiodarone when possible for a week before surgery.

\section{Limitations}

A main limitation of this study is the low number of the patients involved and the low frequency of events. Another limitation is that detailed hemodynamic data were not collected during the perioperative period. The cardiac status of the involved patients in this study may have been better than that of patients receiving long-term amiodarone therapy for treatment of cardiac arrhythmia.

\section{References}

1. Rady MY, Ryan T, Starr NJ. Preoperative therapy with amiodarone and the incidence of acute organ dysfunction after cardiac surgery. Anesth Analg. 1997;85:489-97.

2. Nalos PC, Kass RM, Gang ES, Fishbein MC, Mandel WJ, Peter T. Life-threatening postoperative pulmonary complications in patients with previous amiodarone pulmonary toxicity undergoing cardiothoracic operations. J Thorac Cardiovasc Surg. 1987;93:904-12.

3. Tuzcu EM, Maloney JD, Sangani BH, Masterson ML, Hocevar KD, Golding LA, et al. Cardiopulmonary effects of chronic amiodarone therapy in the early postoperative course of cardiac surgery patients. Cleve Clin J Med. 1987;54:491-7.

4. Kupferschmid JP, Rosengart TK, McIntosh CL, Leon MB, Clark RE. Amiodarone-induced complications after cardiac operation for obstructive hypertrophic cardiomyopathy. Ann Thorac Surg. 1989;48: $359-64$.

5. Greenspon AJ, Kidwell GA, Hurley W, Mannion J. Amiodaronerelated postoperative adult respiratory distress syndrome. Circulation. 1991;84(5 suppl):III407-15.

6. Hawthorne HR, Wood MA, Stambler BS, Damiano RJ, Ellenbogen KA. Can amiodarone pulmonary toxicity be predicted in patients undergoing implantable cardioverter defibrillator implantation? Pacing Clin Electrophysiol. 1993;16:2241-9.

7. Mickleborough LL, Maruyama H, Mohamed S, Rappaport DC, Downar E, Butany J, et al. Are patients receiving amiodarone at increased risk for cardiac operations? Ann Thorac Surg. 1994;58:622-9.

8. Dimopoulou I, Marathias K, Daganou M, Prapas S, Stavridis G, Khoury M, et al. Low-dose amiodarone-related complications after cardiac operations. J Thorac Cardiovasc Surg. 1997;114:31-7.

9. Liberman BA, Teasdale SJ. Anaesthesia and amiodarone. Can Anaesth Soc J. 1985;32:629-38.

10. Terada Y, Mitsui T, Yamaguchi I. Conduction disturbances after open heart operation in a patient receiving amiodarone. Ann Thorac Surg. 1994;58:1213-4.

11. Cairns JA, Connolly SJ, Roberts R, Gent M. Randomised trial of outcome after myocardial infarction in patients with frequent or repetitive ventricular premature depolarisations: CAMIAT. Canadian Amiodarone Myocardial Infarction Arrhythmia Trial Investigators. Lancet. 1997;349:675-82.

12. Mets B, Michler RE, Delphin ED, Oz MC, Landry DW. Refractory vasodilation after cardiopulmonary bypass for heart transplantation in recipients on combined amiodarone and angiotensin- converting enzyme inhibitor therapy: a role for vasopressin administration. J Cardiothorac Vasc Anesth. 1998;12:326-9.

13. Jessurun GA, Boersma WG, Crijns HJ. Amiodarone-induced pulmonary toxicity. Predisposing factors, clinical symptoms and treatment. Drug Saf. 1998;18:339-44.

14. Bharucha DB, Kowey PR. Management and prevention of atrial fibrillation after cardiovascular surgery. Am J Cardiol. 2000;85(10 Suppl 1):20-4. 